PROCEEDINGS OF THE

AMERICAN MATHEMATICAL SOCIETY

Volume 137, Number 3, March 2009, Pages 991-1000

S 0002-9939(08)09691-3

Article electronically published on October 8, 2008

\title{
INCLUSIONS AND COINCIDENCES FOR MULTIPLE SUMMING MULTILINEAR MAPPINGS
}

\author{
G. BOTELHO, H.-A. BRAUNSS, H. JUNEK, AND D. PELLEGRINO \\ (Communicated by Nigel J. Kalton)
}

\begin{abstract}
Using complex interpolation we prove new inclusion and coincidence theorems for multiple (fully) summing multilinear and holomorphic mappings. Among several other results we show that continuous $n$-linear forms on cotype 2 spaces are multiple $\left(2 ; q_{k}, \ldots, q_{k}\right)$-summing, where $2^{k-1}<n \leq 2^{k}$, $q_{0}=2$ and $q_{k+1}=\frac{2 q_{k}}{1+q_{k}}$ for $k \geq 0$.
\end{abstract}

\section{InTRODUCTION AND NOTATION}

The essence of the theory of absolutely summing linear operators can be traced back to Grothendieck's celebrated Resumé [14] and further fundamental works by Pietsch [32] and Lindenstrauss and Pełczyński [19]. For the linear theory of absolutely summing operators the reader is referred to the excellent monograph [11. In 1983 Pietsch 33 . sketched an $n$-linear approach to the theory of absolutely summing operators and since then a vast number of papers has followed this line (e.g., $[1,5,6,8,10,12,13,15,18,21,22,27,28,30,26,29,31,35])$. In this direction, multiple summing (also called fully summing) multilinear mappings were introduced by Matos 21 and, independently, by Bombal, Pérez-García and Villanueva 5. This class has proved to be one of the most useful and fruitful multilinear generalizations of the concept of an absolutely summing linear operator. It is worth mentioning that the bilinear case was first treated in 1985 by Ramanujan and Schock 34. The case of holomorphic mappings is treated in [27]. For the theory of multiple (fully) summing $n$-linear mappings we refer to [5, 21, 28.

In the following, $\mathbb{N}$ denotes the set of all positive integers, $E, E_{1}, \ldots, E_{n}, F$ denote Banach spaces over $\mathbb{K}=\mathbb{R}$ or $\mathbb{C}$. By $E^{\prime}$ we mean the topological dual of $E$ and $B_{E^{\prime}}$ represents its closed unit ball.

Given $n \in \mathbb{N}$, the space of all continuous $n$-linear mappings from $E_{1} \times \cdots \times E_{n}$ to $F$ endowed with the sup norm is denoted by $\mathcal{L}\left(E_{1}, \ldots, E_{n} ; F\right)\left(\mathcal{L}\left({ }^{n} E ; F\right)\right.$ if $E=$ $E_{1}=\cdots=E_{n}$ and $\mathcal{L}(E ; F)$ if $\left.n=1\right)$. The space of all continuous $n$-homogeneous polynomials with the sup norm will be represented by $\mathcal{P}\left({ }^{n} E ; F\right)$. For $p \geq 1$, the vector space of all sequences $\left(x_{j}\right)_{j=1}^{\infty}$ in $E$ such that $\left\|\left(x_{j}\right)_{j=1}^{\infty}\right\|_{p}=\left(\sum_{j=1}^{\infty}\left\|x_{j}\right\|^{p}\right)^{\frac{1}{p}}<$ $\infty$ is denoted by $\ell_{p}(E)$. We represent by $\ell_{p}^{w}(E)$ the linear space of the sequences

Received by the editors March 4, 2008.

2000 Mathematics Subject Classification. Primary 46G25.

The fourth author is supported by CNPq Grant 308084/2006-3 and Edital MCT/CNPq 02/2006-Universal, Grant 471054/2006-2. 
$\left(x_{j}\right)_{j=1}^{\infty}$ in $E$ such that $\left(\varphi\left(x_{j}\right)\right)_{j=1}^{\infty} \in \ell_{p}$ for every $\varphi \in E^{\prime}$. The expression

$$
\left\|\left(x_{j}\right)_{j=1}^{\infty}\right\|_{w, p}:=\sup _{\varphi \in B_{E^{\prime}}}\left\|\left(\varphi\left(x_{j}\right)\right)_{j=1}^{\infty}\right\|_{p}
$$

defines a norm on $\ell_{p}^{w}(E)$. For the corresponding $m$-dimensional spaces we write $\ell_{p}^{m}$ and $\ell_{p, w}^{m}$ instead of $\ell_{p}$ and $\ell_{p}^{w}$ respectively.

Given $1 \leq q_{j} \leq p, j=1, \ldots, n$, an $n$-linear mapping $T: E_{1} \times \cdots \times E_{n} \rightarrow F$ is multiple (or fully) $\left(p ; q_{1}, \ldots, q_{n}\right)$-summing if there exists $C>0$ such that

$$
\left(\sum_{j_{1}, \ldots, j_{n}=1}^{m}\left\|T\left(x_{j_{1}}^{(1)}, \ldots, x_{j_{n}}^{(n)}\right)\right\|^{p}\right)^{1 / p} \leq C \prod_{k=1}^{n}\left\|\left(x_{j}^{(k)}\right)_{j=1}^{m}\right\|_{w, q_{k}}
$$

for every $m \in \mathbb{N}$ and every $x_{j}^{(k)} \in E_{k}, j=1, \ldots, m$ and $k=1, \ldots, n$. The space composed by all multiple $\left(p ; q_{1}, . ., q_{n}\right)$-summing $n$-linear mappings from $E_{1} \times \cdots \times E_{n}$ into $F$ is denoted by $\mathcal{L}_{\operatorname{ms}\left(p ; q_{1}, \ldots, q_{n}\right)}\left(E_{1}, \ldots, E_{n} ; F\right)$, and the infimum of the constants $C$ for which the inequality always holds defines a norm $\|\cdot\|_{\mathrm{ms}\left(p ; q_{1}, \ldots, q_{n}\right)}$ on $\mathcal{L}_{\mathrm{ms}\left(p ; q_{1}, \ldots, q_{n}\right)}\left(E_{1}, \ldots, E_{n} ; F\right)$. If $q_{1}=\cdots=q_{n}=q$, we sometimes write $m s(p ; q)$ instead of $m s(p ; q, \ldots, q)$ and if $p=q=q_{1}=\cdots=q_{n}$ we simply write $m s, p$ instead of $m s(p ; p)$.

An important result due to Bohnenblust and Hille [4 asserts that for each positive integer $n$, there is a constant $c_{n}$ so that

$$
\left(\sum_{j_{1}, \ldots, j_{n}=1}^{\infty}\left|A\left(e_{j_{1}}, \ldots, e_{j_{n}}\right)\right|^{\frac{2 n}{n+1}}\right)^{\frac{n+1}{2 n}} \leq c_{n}\|A\|
$$

for all $A \in \mathcal{L}\left({ }^{n} c_{0} ; \mathbb{K}\right)$. With a simple reformulation of (1.1) one can obtain the "coincidence result"

$$
\mathcal{L}\left(E_{1}, \ldots, E_{n} ; \mathbb{K}\right)=\mathcal{L}_{\operatorname{ms}\left(\frac{2 n}{n+1} ; 1, \ldots, 1\right)}\left(E_{1}, \ldots, E_{n} ; \mathbb{K}\right)
$$

for every $n \geq 2$ and every Banach spaces $E_{1}, \ldots, E_{n}$ (this result appears in [28]). As $\frac{2 n}{n+1} \longrightarrow 2$, it is natural to wonder if multilinear forms are multiple $(2 ; q, \ldots, q)$ summing for some $q>1$. Surprisingly enough we will show that this is true for $n$-linear forms on cotype 2 spaces but with a $q$ depending on $n$. More precisely, in Section 2 we will show that

$$
\mathcal{L}\left(E_{1}, \ldots, E_{n} ; \mathbb{K}\right)=\mathcal{L}_{\operatorname{ms}\left(2 ; q_{k}, \ldots, q_{k}\right)}\left(E_{1}, \ldots, E_{n} ; \mathbb{K}\right),
$$

whenever $E_{1}, \ldots, E_{n}$ have cotype $2,2^{k-1}<n \leq 2^{k}, q_{0}=2$ and $q_{k+1}=\frac{2 q_{k}}{1+q_{k}}$ for $k \geq 0$. Using interpolation techniques, intermediate results are also obtained: if $\theta \in[0,1]$, then

$$
\mathcal{L}\left(E_{1}, \ldots, E_{n} ; \mathbb{K}\right)=\mathcal{L}_{\operatorname{ms}\left(\frac{2 n}{n+\theta}, \frac{q_{k}}{\left(q_{k}-1\right) \theta+1}\right)}\left(E_{1}, \ldots, E_{n} ; \mathbb{K}\right)
$$

for $E_{1}, \ldots, E_{n}, k, n$ and $q_{k}$ as above. These results will be firstly proved for complex Banach spaces and the real case will follow by complexification. As far as we know, this interpolation-complexification argument was first applied to multiple summing mappings by Pérez-García 28. In Section 3 we obtain new inclusions between spaces of multiple summing multilinear, polynomial and holomorphic mappings. 


\section{Coincidence Results FOR MUltiple SUMming FORMS}

Recall that a Banach space $E$ has cotype $q \geq 2$ if there exists a constant $K \geq 0$ such that, no matter how we choose $k \in \mathbb{N}$ and $x_{1}, \ldots, x_{k} \in E$,

$$
\left(\sum_{j=1}^{k}\left\|x_{j}\right\|^{q}\right)^{\frac{1}{q}} \leq K\left(\int_{0}^{1}\left\|\sum_{j=1}^{k} r_{j}(t) x_{j}\right\|^{2} d t\right)^{\frac{1}{2}},
$$

where $r_{j}$ are the Rademacher functions. The infimum of the constants $K$ is denoted by $C_{q}(E)$.

Since $\ell_{2}$ has cotype 2, a particular case of a result obtained (independently) by Pérez-García [28, Teorema 5.2] and Souza [35, Teorema 1.7.3] gives us the following:

Lemma 2.1. For any $n$-tuple $\left(E_{1}, \ldots, E_{n}\right)$ of Banach spaces we have

$$
\mathcal{L}\left(E_{1}, \ldots, E_{n} ; \ell_{2}\right)=\mathcal{L}_{\operatorname{ms}(2 ; 1, \ldots, 1)}\left(E_{1}, \ldots, E_{n} ; \ell_{2}\right) .
$$

Another useful and well-known result that will be useful in the next theorem is the following (the proof is simple, and we omit it):

Lemma 2.2. If $m \geq 1, E_{1}, \ldots, E_{m}, F$ are Banach spaces and

$$
\mathcal{L}\left(E_{1}, \ldots, E_{m} ; F\right)=\mathcal{L}_{\mathrm{ms}(p ; q)}\left(E_{1}, \ldots, E_{m} ; F\right),
$$

then

$$
\mathcal{L}\left(E_{1}, \ldots, E_{n} ; F\right)=\mathcal{L}_{\mathrm{ms}(p ; q)}\left(E_{1}, \ldots, E_{n} ; F\right),
$$

for every $1 \leq n \leq m$.

Henceforth $\left(q_{k}\right)_{k=0}^{\infty}$ will be the sequence of real numbers given by

$$
q_{0}=2 \text { and } q_{k+1}=\frac{2 q_{k}}{1+q_{k}} \text { for } k \geq 0 .
$$

Theorem 2.3. Let $n \geq 1$ and let $E_{1}, \ldots, E_{n}$ be Banach spaces of cotype 2 . If $k$ is the natural number such that $2^{k-1}<n \leq 2^{k}$, then

$$
\mathcal{L}\left(E_{1}, \ldots, E_{n} ; \mathbb{K}\right)=\mathcal{L}_{\mathrm{ms}\left(2 ; q_{k}, \ldots, q_{k}\right)}\left(E_{1}, \ldots, E_{n} ; \mathbb{K}\right) .
$$

Proof. First we prove the complex case $\mathbb{K}=\mathbb{C}$. We can assume that $n=2^{k}$ for some natural $k \geq 0$, because otherwise we could choose a natural $k$ such that $n<2^{k}$ and extend the $n$-tuple $\left(E_{1}, \ldots, E_{n}\right)$ to the $2^{k}$-tuple $\left(E_{1}, \ldots, E_{n}, \mathbb{C}, \ldots, \mathbb{C}\right)$ and use Lemma 2.2. We are going to prove the claim by induction over $k$. For $k=0$ there is nothing left to do. Suppose now that the claim is true for $n=2^{k}$. Let us consider any $2 n$-linear form $T \in \mathcal{L}\left(E_{1}, \ldots, E_{n}, F_{1}, \ldots, F_{n} ; \mathbb{C}\right)$ with spaces $E_{i}, F_{j}$ of cotype 2 $\left(2^{k+1}=2 n\right)$. In a first step we are going to show that

$$
T \in \mathcal{L}_{\operatorname{ms}\left(2 ; 1, \ldots, 1, q_{k}, \ldots, q_{k}\right)}\left(E_{1}, \ldots, E_{n}, F_{1}, \ldots, F_{n} ; \mathbb{C}\right) .
$$

For fixed $m \geq 1$ and all $1 \leq r, s \leq n=2^{k}$ let any $m$-tuples

$$
\left(x_{i_{r}}^{(r)}\right)_{i_{r}=1}^{m} \subset E_{r} \text { and }\left(y_{j_{s}}^{(s)}\right)_{j_{s}=1}^{m} \subset F_{s}
$$

be given. For the sake of abbreviation we put

$\mathbf{x}_{\mathbf{i}}=\left(x_{i_{1}}^{(1)}, \ldots, x_{i_{n}}^{(n)}\right)$ for $\mathbf{i}=\left(i_{1}, \ldots, i_{n}\right)$ and $\mathbf{y}_{\mathbf{j}}=\left(y_{j_{1}}^{(1)}, \ldots, y_{j_{n}}^{(n)}\right)$ for $\mathbf{j}=\left(j_{1}, \ldots, j_{n}\right)$.

For fixed $\mathbf{y}_{\mathbf{j}}$ we define

$$
T_{\mathbf{y}_{\mathbf{j}}} \in \mathcal{L}\left(E_{1}, \ldots, E_{n} ; \mathbb{C}\right) \text { by } T_{\mathbf{y}_{\mathbf{j}}}\left(x_{1}, \ldots, x_{n}\right)=T\left(x_{1}, \ldots, x_{n}, \mathbf{y}_{\mathbf{j}}\right) .
$$


By the induction assumption we have that $T_{\mathbf{y}_{\mathbf{j}}} \in \mathcal{L}_{\mathrm{ms}\left(2, q_{k}, \ldots, q_{k}\right)}\left(E_{1}, \ldots, E_{n} ; \mathbb{C}\right)$. Now define

$$
S \in \mathcal{L}\left(E_{1}, \ldots, E_{n} ; \ell_{2}\right) \text { by } S \mathbf{x}=\left(T\left(\mathbf{x}, \mathbf{y}_{\mathbf{j}}\right)_{\mathbf{j} \in\{1, \ldots, m\}^{n}}, 0, \ldots\right) \in \ell_{2} .
$$

Lemma 2.1 gives that $S \in \mathcal{L}_{\mathrm{ms}(2 ; 1, \ldots, 1)}\left(E_{1}, \ldots, E_{n} ; \ell_{2}\right)$, i.e.

$$
\left(\sum_{\mathbf{i}}\left\|S \mathbf{x}_{\mathbf{i}}\right\|^{2}\right)^{1 / 2} \leq c\|S\| \cdot \prod_{r=1}^{n}\left\|\left(x_{j_{r}}^{(r)}\right)_{j_{r}=1}^{m}\right\|_{w, 1} .
$$

Further, from the induction assumption there is $c_{1}$ such that $\|T(\mathbf{x}, \cdot)\|_{\operatorname{ms}\left(2 ; q_{k}, \ldots, q_{k}\right)} \leq$ $c_{1}\|T(\mathbf{x}, \cdot)\|$ for every $\mathbf{x} \in E_{1} \times \cdots \times E_{n}$. So,

$$
\begin{aligned}
\|S\| & =\sup _{\mathbf{x} \in B_{E_{1}} \times \cdots \times B_{E_{n}}}\|S \mathbf{x}\|_{2}=\sup _{\mathbf{x} \in B_{E_{1}} \times \cdots \times B_{E_{n}}}\left(\sum_{\mathbf{j}}\left|T\left(\mathbf{x}, \mathbf{y}_{\mathbf{j}}\right)\right|^{2}\right)^{1 / 2} \\
& \leq c_{1} \sup _{\mathbf{x} \in B_{E_{1}} \times \cdots \times B_{E_{n}}}\|T(\mathbf{x}, \cdot)\| \prod_{s=1}^{n}\left\|\left(y_{j_{s}}^{(s)}\right)_{j_{s}=1}^{m}\right\|_{w, q_{k}} \leq c_{1}\|T\| \prod_{s=1}^{n}\left\|\left(y_{j_{s}}^{(s)}\right)_{j_{s}=1}^{m}\right\|_{w, q_{k}} .
\end{aligned}
$$

Plugging this into (2.2) we end up with

$$
\begin{aligned}
\left(\sum_{\mathbf{i}} \sum_{\mathbf{j}} \mid\right. & \left.\left.T\left(\mathbf{x}_{\mathbf{i}}, \mathbf{y}_{\mathbf{j}}\right)\right|^{2}\right)^{1 / 2} \\
& =\left(\sum_{\mathbf{i}}\left\|S \mathbf{x}_{\mathbf{i}}\right\|^{2}\right)^{1 / 2} \leq c_{2} \cdot \prod_{r=1}^{n}\left\|\left(x_{j_{r}}^{(r)}\right)_{j_{r}=1}^{m}\right\|_{w, 1} \cdot \prod_{s=1}^{n}\left\|\left(y_{j_{s}}^{(s)}\right)_{j_{s}=1}^{m}\right\|_{w, q_{k}},
\end{aligned}
$$

which proves (2.1). By symmetry we also have

$$
T \in \mathcal{L}_{\operatorname{ms}\left(2 ; q_{k}, \ldots, q_{k}, 1, \ldots, 1\right)}\left(E_{1}, \ldots, E_{n}, F_{1}, \ldots, F_{n} ; \mathbb{C}\right) .
$$

We proceed by complex interpolation. It follows from (2.1) and (2.3) that the $2 n$-linear mappings

$$
\begin{aligned}
& \Psi_{T}^{(0)}: \ell_{1, w}^{m}\left(E_{1}\right) \times \cdots \times \ell_{1, w}^{m}\left(E_{n}\right) \times \ell_{q_{k}, w}^{m}\left(F_{1}\right) \times \cdots \times \ell_{q_{k}, w}^{m}\left(F_{n}\right) \rightarrow \ell_{2}^{m^{2 n}}(\mathbb{C}), \\
& \Psi_{T}^{(1)}: \ell_{q_{k}, w}^{m}\left(E_{1}\right) \times \cdots \times \ell_{q_{k}, w}^{m}\left(E_{n}\right) \times \ell_{1, w}^{m}\left(F_{1}\right) \times \cdots \times \ell_{1, w}^{m}\left(F_{n}\right) \rightarrow \ell_{2}^{m^{2 n}}(\mathbb{C})
\end{aligned}
$$

given by

$$
\begin{aligned}
& \left(\left(x_{i_{1}}^{(1)}\right)_{i_{1}}, \ldots,\left(x_{i_{n}}^{(n)}\right)_{i_{n}},\left(y_{j_{1}}^{(1)}\right)_{j_{1}}, \ldots,\left(y_{j_{n}}^{(n)}\right)_{j_{n}}\right) \mapsto \\
& \left(T\left(x_{i_{1}}^{(1)}, \ldots, x_{i_{n}}^{(n)}, y_{j_{1}}^{(1)}, \ldots, y_{j_{n}}^{(n)}\right)\right)_{i_{1}, \ldots, i_{n}, j_{1}, \ldots, j_{n}=1}^{m}
\end{aligned}
$$

are bounded independently of $m$ by

$$
\left\|\Psi_{T}^{(0)}\right\| \leq\|T\|_{\mathrm{ms}\left(2 ; 1, \ldots, 1, q_{k}, \ldots, q_{k}\right)}:=K_{0} \text { and }\left\|\Psi_{T}^{(1)}\right\| \leq\|T\|_{\mathrm{ms}\left(2 ; q_{k}, \ldots, q_{k}, 1, \ldots, 1\right)}:=K_{1},
$$

respectively. Remember that for any Banach space $G$ and any $1 \leq s<\infty$ there is a natural linear isometry between $\ell_{s, w}^{m}(G)$ and the injective tensor product $\ell_{s}^{m} \otimes_{\varepsilon} G$. Therefore, $\Psi_{T}^{(0)}$ and $\Psi_{T}^{(1)}$ can also be considered as mappings on the Cartesian product of the associated tensor products with the same operator norm. Using 
complex multilinear interpolation [2, Theorem 4.4.1] for $\theta=1 / 2$, we obtain a $2 n$ multilinear operator

$$
\begin{aligned}
\Psi_{T}^{(1 / 2)}:\left[\ell_{1}^{m} \otimes_{\varepsilon} E_{1}, \ell_{q_{k}}^{m}\right. & \left.\otimes_{\varepsilon} E_{1}\right]_{1 / 2} \times \cdots \times\left[\ell_{q_{k}}^{m} \otimes_{\varepsilon} F_{1}, \ell_{1}^{m} \otimes_{\varepsilon} F_{1}\right]_{1 / 2} \times \cdots \\
& \rightarrow\left[\ell_{2}^{m^{2 n}}(\mathbb{C}), \ell_{2}^{m^{2 n}}(\mathbb{C})\right]_{1 / 2}
\end{aligned}
$$

with $\left\|\Psi_{T}^{(1 / 2)}\right\| \leq K_{0}^{1 / 2} K_{1}^{1 / 2}$. Now the interpolation result due to Defant and Michels 9] for $\varepsilon$-tensor products comes into play. Since $\ell_{q}$ is $q$-concave for $1 \leq q \leq 2$, we conclude by [9, Theorem, p. 441] (which is an extension of a classical result due to Kouba [17, Theorem 4.2.11]) that

$$
\left[\ell_{1}^{m} \otimes_{\varepsilon} G, \ell_{q}^{m} \otimes_{\varepsilon} G\right]_{1 / 2}=\left[\ell_{1}^{m}, \ell_{q}^{m}\right]_{1 / 2} \otimes_{\varepsilon} G=\ell_{p}^{m} \otimes_{\varepsilon} G
$$

with isomorphism constants not depending on $m$, provided that $G$ has cotype 2, $1 \leq q \leq 2$ and $\frac{1}{p}=\frac{1 / 2}{1}+\frac{1 / 2}{q}$. So, $\Psi_{T}^{(1 / 2)}$ can also be considered as a map

$$
\Psi_{T}^{(1 / 2)}: \ell_{q_{k+1}}^{m} \otimes_{\varepsilon} E_{1} \times \cdots \times \ell_{q_{k+1}}^{m} \otimes_{\varepsilon} F_{n} \rightarrow \ell_{2}^{m^{2 n}}
$$

with $\left\|\Psi_{T}^{(1 / 2)}\right\| \leq c_{3} \cdot K_{0}^{1 / 2} K_{1}^{1 / 2}$ for some constant $c_{3}$ not depending on $m$ and $\frac{1}{q_{k+1}}=\frac{1 / 2}{1}+\frac{1 / 2}{q_{k}}$, i.e. $q_{k+1}=\frac{2 q_{k}}{q_{k}+1}$. In terms of $T$ this means that

$$
\left(\sum_{\mathbf{i}} \sum_{\mathbf{j}}\left|T\left(\mathbf{x}_{\mathbf{i}}, \mathbf{y}_{\mathbf{j}}\right)\right|^{2}\right)^{1 / 2} \leq c_{4} \cdot \prod_{r=1}^{n}\left\|\left(x_{j_{r}}^{(r)}\right)_{j_{r}=1}^{m}\right\|_{w, q_{k+1}} \cdot \prod_{s=1}^{n}\left\|\left(y_{j_{s}}^{(s)}\right)_{j_{s}=1}^{m}\right\|_{w, q_{k+1}}
$$

with some constant $c_{4}$ not depending on $m$, and so the complex case is done. To prove the real case we proceed by complexification. Given real Banach spaces $E_{1}, \ldots, E_{n}$ of cotype 2 and $T \in \mathcal{L}\left(E_{1}, \ldots, E_{n} ; \mathbb{R}\right)$, by $\widetilde{E_{1}}, \ldots, \widetilde{E_{n}}$ we mean their respective complexifications (see 23,24$]$ ) and by $\tilde{T} \in \mathcal{L}\left(\widetilde{E_{1}}, \ldots, \widetilde{E_{n}} ; \mathbb{C}\right)$ the extension of $T$ according to [3, Theorem 3]. By [28, Proposición 4.30(ii)] we know that $\widetilde{E_{1}}, \ldots, \widetilde{E_{n}}$ have cotype 2 , so the first part of the proof yields that $\tilde{T}$ is multiple $\left(2 ; q_{k}, \ldots, q_{k}\right)$-summing. It follows from (an easy adaptation of) 28, Proposición $4.30(\mathrm{i})]$ that $T$ is multiple $\left(2 ; q_{k}, \ldots, q_{k}\right)$-summing as well.

Now we obtain a scale of coincidences from (1.2) to Theorem 2.3:

Theorem 2.4. Let $n \geq 1$ and let $E_{1}, \ldots, E_{n}$ be Banach spaces of cotype 2 . If $k$ is the natural number such that $2^{k-1}<n \leq 2^{k}$, then

$$
\mathcal{L}\left(E_{1}, \ldots, E_{n} ; \mathbb{K}\right)=\mathcal{L}_{\operatorname{ms}\left(\frac{2 n}{n+\theta} ; \frac{q_{k}}{\left(q_{k}-1\right) \theta+1}\right)}\left(E_{1}, \ldots, E_{n} ; \mathbb{K}\right)
$$

for every $\theta \in[0,1]$.

Proof. By (1.2) and Theorem 2.3 we know that

$$
\begin{aligned}
& \mathcal{L}\left(E_{1}, \ldots, E_{n} ; \mathbb{C}\right)=\mathcal{L}_{\mathrm{ms}\left(\frac{2 n}{n+1} ; 1,1, \ldots, 1\right)}\left(E_{1}, \ldots, E_{n} ; \mathbb{C}\right) \text { and } \\
& \mathcal{L}\left(E_{1}, \ldots, E_{n} ; \mathbb{C}\right)=\mathcal{L}_{\operatorname{ms}\left(2 ; q_{k}, \ldots, q_{k}\right)}\left(E_{1}, \ldots, E_{n} ; \mathbb{C}\right) .
\end{aligned}
$$

Since

$$
\frac{1}{\frac{2 n}{n+\theta}}=\frac{1-\theta}{2}+\frac{\theta}{\frac{2 n}{n+1}} \text { and } \frac{1}{\frac{q_{k}}{\left(q_{k}-1\right) \theta+1}}=\frac{1-\theta}{q_{k}}+\frac{\theta}{1},
$$

the same interpolation-complexification argument furnishes the result. 


\section{INCLUSION RESULTS}

Given $1 \leq p \leq q<\infty$, it is well known that absolutely $p$-summing linear operators are absolutely $q$-summing. For multiple summing mappings, Pérez-García [28. Teorema 4.13] has shown that $\mathcal{L}_{\mathrm{ms}, p}\left(E_{1}, \ldots, E_{n} ; F\right) \subseteq \mathcal{L}_{\mathrm{ms}, q}\left(E_{1}, \ldots, E_{n} ; F\right)$ whenever $1 \leq p \leq q<2\left(1 \leq p \leq q \leq 2\right.$ if $E_{1}, \ldots, E_{n}$ have cotype 2$)$. However, in [28. Teorema 4.13 ] it is also shown that there is no general inclusion theorem for multiple summing multilinear mappings. Some surprising inclusion results for absolutely summing polynomials and holomorphic mappings were recently obtained in [16. In this section we obtain new inclusions for multiple summing multilinear mappings, polynomials and holomorphic mappings.

A result obtained independently by Pérez-García [28, Teorema 5.2] and Souza [35, Teorema 1.7.3] asserts that if $F$ has finite cotype $q$, then

$$
\mathcal{L}_{\operatorname{ms}(q ; 1)}\left({ }^{n} E ; F\right)=\mathcal{L}\left({ }^{n} E ; F\right) \text { and }\|\cdot\|_{\operatorname{ms}(q ; 1)} \leq C_{q}(F)^{n}\|\cdot\| \text {. }
$$

Next we will show how (3.1) can be explored in order to obtain surprising inclusion results. For the complexification argument to work we need the following extension of [28, Proposición 4.30(ii)]:

Lemma 3.1. A real Banach space $E$ has cotype $q>2$ if and only if its complexification $\widetilde{E}$ has cotype $q>2$. Also, if $E$ has cotype 2, then $\widetilde{E}$ has cotype 2.

Proof. The cotype 2 case is done in [28, Proposición 4.30(ii)]. Assume $q>2$. A celebrated result due to Talagrand [36] asserts that a Banach space $E$ has cotype $q$ if and only if $i d_{E}$ is absolutely $(q ; 1)$-summing. So, from the linear case of [28, Proposición 4.30(ii)] we have that

$$
\begin{aligned}
E \text { has cotype } q & \Leftrightarrow i d_{E} \text { is }(q ; 1) \text {-summing } \\
& \Leftrightarrow i d_{\widetilde{E}} \text { is }(q ; 1) \text {-summing } \Leftrightarrow \widetilde{E} \text { has cotype } q .
\end{aligned}
$$

Remember that whenever we write $\operatorname{ms}(r ; s)$ we are assuming $1 \leq s \leq r$.

Theorem 3.2. If $E_{1}, \ldots, E_{n}$ have cotype $2, F$ has finite cotype $q$ and $1 \leq s \leq 2$, then

$$
\mathcal{L}_{\mathrm{ms}(r ; s)}\left(E_{1}, \ldots, E_{n} ; F\right) \subseteq \mathcal{L}_{\mathrm{ms}\left(t_{1} ; t_{2}\right)}\left(E_{1}, \ldots, E_{n} ; F\right)
$$

for every $n \in \mathbb{N}, 0 \leq \theta \leq 1$ and $t_{1}, t_{2}$ satisfying

$$
\frac{1}{t_{1}}=\frac{1-\theta}{r}+\frac{\theta}{q} \text { and } \frac{1}{t_{2}}=\frac{1-\theta}{s}+\theta .
$$

Moreover, if $T \in \mathcal{L}_{\mathrm{ms}(r ; s)}\left(E_{1}, \ldots, E_{n} ; F\right)$, then

$$
\|T\|_{\mathrm{ms}\left(t_{1} ; t_{2}\right)} \leq 16^{n}\left(C_{2}\left(E_{1}\right) \cdots C_{2}\left(E_{n}\right)\right)^{\frac{5}{2}} C_{q}(F)^{n \theta}\|T\|_{\mathrm{ms}(r ; s)}^{(1-\theta)}\|T\|^{\theta} .
$$

Proof. As before, using [28, Proposición 4.30(ii)] and Lemma 3.1, the real case follows from the complex case. Assume $\mathbb{K}=\mathbb{C}$.

Claim. Under the assumptions of the theorem, if $T \in \mathcal{L}_{\mathrm{ms}(r ; s)}\left(E_{1}, \ldots, E_{n} ; F\right) \cap$ $\mathcal{L}_{\mathrm{ms}(p ; h)}\left(E_{1}, \ldots, E_{n} ; F\right)$ for some $1 \leq h \leq 2$, then $T \in \mathcal{L}_{\mathrm{ms}\left(t_{1} ; t_{2}\right)}\left(E_{1}, \ldots, E_{n} ; F\right)$ and

$$
\|T\|_{\mathrm{ms}\left(t_{1} ; t_{2}\right)} \leq 16^{n}\left(C_{2}\left(E_{1}\right) \cdots C_{2}\left(E_{n}\right)\right)^{\frac{5}{2}}\|T\|_{\mathrm{ms}(r ; s)}^{1-\theta}\|T\|_{\mathrm{ms}(p ; h)}^{\theta}
$$


where $\theta \in[0,1]$ and $t_{1}, t_{2}$ satisfy

$$
\frac{1}{t_{1}}=\frac{1-\theta}{r}+\frac{\theta}{p} \text { and } \frac{1}{t_{2}}=\frac{1-\theta}{s}+\frac{\theta}{h} .
$$

Proof of the claim: We proceed by complex interpolation. For each positive integer $m$ the map $T$ induces natural (uniformly) bounded $n$-linear mappings

$$
\begin{aligned}
& \Psi_{T}^{(a)}: \ell_{s, w}^{m}\left(E_{1}\right) \times \cdots \times \ell_{s, w}^{m}\left(E_{n}\right) \rightarrow \ell_{r}^{m^{n}}(F) \text { and } \\
& \Psi_{T}^{(b)}: \ell_{h, w}^{m}\left(E_{1}\right) \times \cdots \times \ell_{h, w}^{m}\left(E_{n}\right) \rightarrow \ell_{p}^{m^{n}}(F) .
\end{aligned}
$$

Applying the complex interpolation method [2, Theorem 4.4.1] to these $n$-linear operators we get an $n$-linear mapping

$$
\Psi_{T}^{(\theta)}:\left[\ell_{s, w}^{m}\left(E_{1}\right), \ell_{h, w}^{m}\left(E_{1}\right)\right]_{\theta} \times \cdots \times\left[\ell_{s, w}^{m}\left(E_{n}\right), \ell_{h, w}^{m}\left(E_{n}\right)\right]_{\theta} \rightarrow\left[\ell_{r}^{m^{n}}(F), \ell_{p}^{m^{n}}(F)\right]_{\theta}
$$

with $\left\|\Psi_{T}^{(\theta)}\right\| \leq\left\|\Psi_{T}^{(a)}\right\|^{1-\theta}\left\|\Psi_{T}^{(b)}\right\|^{\theta}$. By [2, Theorem 5.1.2] we have the isometry

$$
\left[\ell_{r}^{m^{n}}(F), \ell_{p}^{m^{n}}(F)\right]_{\theta}=\ell_{t_{1}}^{m^{n}}(F)
$$

with $t_{1}$ as in (3.3). Using the natural isometric identification $\ell_{s, w}^{m}\left(E_{k}\right)=\ell_{s}^{m} \otimes_{\varepsilon} E_{k}$, $k=1, \ldots, n$, as a particular case of [9, Lemma 2 and Proposition 8] (remember that $\ell_{s}$ and $\ell_{h}$ are 2-concave with constant 1 because $s, h \in[1,2]$ ), we obtain natural isomorphisms

$$
J_{k}: \ell_{t_{2}, w}^{m}\left(E_{k}\right) \rightarrow\left[\ell_{s, w}^{m}\left(E_{k}\right), \ell_{h, w}^{m}\left(E_{k}\right)\right]_{\theta}
$$

with $t_{2}$ as in (3.3) and $\left\|J_{k}\right\| \leq 16 C_{2}\left(E_{k}\right)^{\frac{5}{2}}$. Up to these isomorphisms the mapping $\Psi_{T}^{(\theta)}$ can be identified with the multilinear mapping

$$
\begin{gathered}
\Psi_{T}: \ell_{t_{2}, w}^{m}\left(E_{1}\right) \times \cdots \times \ell_{t_{2}, w}^{m}\left(E_{n}\right) \rightarrow \ell_{t_{1}}^{m^{n}}(F), \\
\Psi_{T}\left(\left(x_{j}^{(1)}\right)_{j=1}^{m}, \ldots,\left(x_{j}^{(n)}\right)_{j=1}^{m}\right)=\left(T\left(x_{j_{1}}^{(1)}, \ldots, x_{j_{n}}^{(n)}\right)\right)_{j_{1}, \ldots, j_{n}=1}^{m}
\end{gathered}
$$

for all sequences $\left(x_{j}^{(k)}\right)_{j=1}^{m}$ in $\ell_{t_{2}, w}^{m}\left(E_{k}\right)=\left[\ell_{s, w}^{m}\left(E_{k}\right), \ell_{h, w}^{m}\left(E_{k}\right)\right]_{\theta}, 1 \leq k \leq n$. This gives us that $T \in \mathcal{L}_{\mathrm{ms}\left(t_{1} ; t_{2}\right)}\left(E_{1}, \ldots, E_{n} ; F\right)$ and

$$
\begin{aligned}
\|T\|_{\mathrm{ms}\left(t_{1}, t_{2}\right)} & \leq\left\|J_{1}\right\| \cdots\left\|J_{n}\right\|\left\|\Psi_{T}^{(\theta)}\right\| \leq 16^{n}\left(C_{2}\left(E_{1}\right) \cdots C_{2}\left(E_{n}\right)\right)^{\frac{5}{2}}\left\|\Psi_{T}^{(a)}\right\|^{1-\theta}\left\|\Psi_{T}^{(b)}\right\|^{\theta} \\
& \leq 16^{n}\left(C_{2}\left(E_{1}\right) \cdots C_{2}\left(E_{n}\right)\right)^{\frac{5}{2}}\|T\|_{\mathrm{ms}(r ; s)}^{1-\theta}\|T\|_{\mathrm{ms}(p ; h)}^{\theta},
\end{aligned}
$$

which proves the claim.

To get the result just make $p=q$ and $h=1$ in the claim and call on (3.1).

Remark 3.3. Theorem 3.2 is interesting for $r \geq q$. The case $r<q$ is trivial.

Example 3.4. Under the hypotheses of Theorem 3.2 we have

$$
\mathcal{L}_{\mathrm{ms}(r ; s)}\left({ }^{n} E ; F\right) \subseteq \mathcal{L}_{\operatorname{ms}\left(\frac{q r}{(1-\theta) q+\theta r} ; \frac{s}{(1-\theta)+\theta s}\right)}\left({ }^{n} E ; F\right),
$$

which can be regarded as a multilinear version (under certain additional hypotheses) of [11, Theorem 10.4]. For instance, making $r=4, s=2, q=3, \theta=1 / 2, E=\ell_{2}$ and $F=\ell_{3}$, we obtain

$$
\mathcal{L}_{\mathrm{ms}(4 ; 2)}\left({ }^{n} \ell_{2} ; \ell_{3}\right) \subseteq \mathcal{L}_{\mathrm{ms}\left(\frac{24}{7} ; \frac{4}{3}\right)}\left({ }^{n} \ell_{2} ; \ell_{3}\right)
$$

for every positive integer $n$. 
We finish the paper by showing how Theorem 3.2 can be applied to multiple summing homogeneous polynomials and holomorphic mappings.

Recall that an $n$-homogeneous polynomial $P: E \rightarrow F$ is multiple (or fully) $(r ; s)$ summing (in symbols $P \in \mathcal{P}_{\operatorname{ms}(r ; s)}\left({ }^{n} E ; F\right)$ ) if its associated symmetric $n$-linear mapping $\stackrel{\vee}{P}$ is multiple $(r ; s)$-summing. A natural norm on $\mathcal{P}_{\operatorname{ms}(r ; s)}\left({ }^{n} E ; F\right)$ is given by $\|P\|_{\mathrm{ms}(r ; s)}=\|\stackrel{\vee}{P}\|_{\mathrm{ms}(r ; s)}$. It is well known [27, Theorem 4.3] that $\left(\mathcal{P}_{\mathrm{ms}(p ; q)},\|\cdot\|_{\mathrm{ms}(p ; q)}\right)$ is a (global) holomorphy type (for the definition and further details on global holomorphy types the reader is referred to [7]).

From Theorem 3.2 and the estimate $\|\stackrel{\vee}{P}\| \leq e^{n}\|P\|$ we obtain:

Proposition 3.5. If $E$ has cotype $2, F$ has finite cotype $q$, and $1 \leq s \leq 2$, then

$$
\mathcal{P}_{\mathrm{ms}(r ; s)}\left({ }^{n} E ; F\right) \subseteq \mathcal{P}_{\operatorname{ms}\left(t_{1} ; t_{2}\right)}\left({ }^{n} E ; F\right)
$$

for every $0 \leq \theta \leq 1$ and $t_{1}, t_{2}$ satisfying

$$
\frac{1}{t_{1}}=\frac{1-\theta}{r}+\frac{\theta}{q} \text { and } \frac{1}{t_{2}}=\frac{1-\theta}{s}+\theta .
$$

Moreover, if $P \in \mathcal{P}_{\operatorname{ms}(r, s)}\left({ }^{n} E ; F\right)$, then

$$
\|P\|_{\mathrm{ms}\left(t_{1}, t_{2}\right)} \leq\left(16 e^{\theta}\right)^{n} C_{2}(E)^{\frac{5 n}{2}} C_{q}(F)^{n \theta}\|P\|_{\mathrm{ms}(r, s)}^{(1-\theta)}\|P\|^{\theta} .
$$

Definition 3.6. An entire mapping $f: E \rightarrow F$ is said to be of $\operatorname{ms}(p ; q)$-holomorphy type at $a \in E$ (in the sense of Nachbin [25]) if

(a) $\frac{1}{n !} \widehat{d}^{n} f(a) \in \mathcal{P}_{\operatorname{ms}(p ; q)}\left({ }^{n} E ; F\right)$ and

(b) there exist $C_{1} \geq 0$ and $c_{1} \geq 0$ such that

$$
\left\|\frac{1}{n !} \widehat{d}^{n} f(a)\right\|_{\operatorname{ms}(p ; q)} \leq C_{1} c_{1}^{n}
$$

for every positive integer $n$. If $f$ is of $\operatorname{ms}(p ; q)$-holomorphy type at every $a \in E$, we say that $f$ is of $\operatorname{ms}(p ; q)$-holomorphy type and we write $f \in \mathcal{H}_{\mathrm{ms}(p ; q)}(E ; F)$. The following inclusion follows immediately from [28, Teorema 4.13]:

Proposition 3.7. If $1 \leq p<q<2$ and $E, F$ are complex Banach spaces, then $\mathcal{H}_{\mathrm{ms}, p}(E ; F) \subseteq \mathcal{H}_{\mathrm{ms}, q}(E ; F)$.

To holomorphic mappings of $\mathrm{ms}(p ; q)$-holomorphy type we have the following extension of Proposition 3.5 .

Proposition 3.8. Let $E, F$ be complex Banach spaces. If $E$ has cotype $2, F$ has finite cotype $q$, and $1 \leq s \leq 2$, then

$$
\mathcal{H}_{\mathrm{ms}(r ; s)}(E ; F) \subseteq \mathcal{H}_{\mathrm{ms}\left(t_{1} ; t_{2}\right)}(E ; F)
$$

for every $0 \leq \theta \leq 1$ and $t_{1}, t_{2}$ satisfying

$$
\frac{1}{t_{1}}=\frac{1-\theta}{r}+\frac{\theta}{q} \text { and } \frac{1}{t_{2}}=\frac{1-\theta}{s}+\theta .
$$

Proof. Let $f \in \mathcal{H}_{\mathrm{ms}(r ; s)}(E ; F)$ and $a \in E$. Then $\frac{1}{n !} \widehat{d}^{n} f(a) \in \mathcal{P}_{\operatorname{ms}(r ; s)}\left({ }^{n} E ; F\right)$ and there are positive constants $C_{1}, c_{1}, C$ and $c$ so that

$$
\left\|\frac{1}{n !} \widehat{d}^{n} f(a)\right\| \leq C_{1} c_{1}^{n} \text { and }\left\|\frac{1}{n !} \widehat{d}^{n} f(a)\right\|_{\mathrm{ms}(r ; s)} \leq C c^{n}
$$


for all $n$. From (3.4) we conclude that $\frac{1}{n !} \widehat{d}^{n} f(a) \in \mathcal{P}_{\mathrm{ms}\left(t_{1} ; t_{2}\right)}\left({ }^{n} E ; F\right)$, and from (3.5) we get

$$
\begin{aligned}
\left\|\frac{1}{n !} \widehat{d}^{n} f(a)\right\|_{\mathrm{ms}\left(t_{1} ; t_{2}\right)} & \leq\left(16 e^{\theta}\right)^{n} C_{2}(E)^{\frac{5 n}{2}} C_{q}(F)^{n \theta}\left\|\frac{1}{n !} \widehat{d}^{n} f(a)\right\|_{\mathrm{ms}(r ; s)}^{(1-\theta)}\left\|\frac{1}{n !} \widehat{d}^{n} f(a)\right\|^{\theta} \\
& \leq\left(16 e^{\theta} C_{2}(E)^{\frac{5}{2}} C_{q}(F)^{\theta}\right)^{n}\left(C c^{n}\right)^{1-\theta}\left(C_{1} c_{1}^{n}\right)^{\theta} \\
& =C^{1-\theta} C_{1}^{\theta}\left(16 e^{\theta} C_{2}(E)^{\frac{5}{2}} C_{q}(F)^{\theta} c_{1}^{\theta} c^{1-\theta}\right)^{n}
\end{aligned}
$$

which shows that $f \in \mathcal{H}_{\mathrm{ms}\left(t_{1} ; t_{2}\right)}(E ; F)$.

\section{ACKNOWLEDGEMENT}

The authors thank the referee for helpful suggestions.

\section{REFERENCES}

[1] M. Acosta, D. García and M. Maestre, A multilinear Lindenstrauss theorem, J. Funct. Anal. 235 (2006), 122-136. MR2216442 (2007b:46065)

[2] J. Bergh and J. Löfström, Interpolation spaces. An introduction, Springer-Verlag, BerlinNew York, 1976. MR0482275 (58:2349)

[3] J. Bochnak and J. Siciak, Polynomials and multilinear mappings in topological vector spaces, Studia Math. 39 (1971), 59-76. MR0313810(47:2364)

[4] H. F. Bohnenblust and E. Hille, On the absolute convergence of Dirichlet series, Ann. of Math. (2) 32 (1931), 600-622. MR 1503020

[5] F. Bombal, D. Pérez-García and I. Villanueva, Multilinear extensions of Grothendieck's theorem, Quart. J. Math. 55 (2004), 441-450. MR2104683 (2005i:47032)

[6] G. Botelho, Cotype and absolutely summing multilinear mappings and homogeneous polynomials, Proc. Roy. Irish Acad. Sect. A 97 (1997), 145-153. MR1645283 (99i:46006)

[7] G. Botelho, H.-A. Braunss, H. Junek and D. Pellegrino, Holomorphy types and ideals of multilinear mappings, Studia Math. 177 (2006), 43-65. MR.2283707 (2008a:46046)

[8] G. Botelho and D. Pellegrino, Scalar-valued dominated polynomials on Banach spaces, Proc. Amer. Math. Soc. 134 (2006), 1743-1751. MR2204287 (2006i:46063)

[9] A. Defant and C. Michels, A complex interpolation formula for tensor products of vectorvalued Banach function spaces, Arch. Math. 74 (2000), 441-451. MR.1753543 (2001d:46103)

[10] A. Defant and D. Pérez-García, A tensor norm preserving unconditionality for $\mathcal{L}_{p}$-spaces, Trans. Amer. Math. Soc. 360 (2008), 3287-3306. MR2379797

[11] J. Diestel, H. Jarchow and A. Tonge, Absolutely summing operators, Cambridge University Press, 1995. MR $1342297(96 \mathrm{i}: 46001)$

[12] V. Dimant, Strongly p-summing multilinear operators, J. Math. Anal. Appl. 278 (2003), 182-193. MR1963473 (2003m:47031)

[13] S. Geiss, Ein Faktorisierungssatz für multilineare Funktionale, Math. Nachr. 134 (1987), 149-159. MR918674 (89b:47067)

[14] A. Grothendieck, Résumé de la théorie métrique des produits tensoriels topologiques, Boletim da Sociedade Matemática de São Paulo 8 (1953), 1-79. MR0094682 (20:1194)

[15] H. Jarchow, C. Palazuelos, D. Pérez-García and I. Villanueva, Hahn-Banach extension of multilinear forms and summability, J. Math. Anal. Appl. 336 (2007), 1161-1177. MR2353008

[16] H. Junek, M. C. Matos and D. Pellegrino, Inclusion theorems for absolutely summing holomorphic mappings, Proc. Amer. Math. Soc. 136 (2008), 3983-3991.

[17] O. Kouba, On the interpolation of injective or projective tensor products of Banach spaces, J. Funct. Anal. 96 (1991), 38-61. MR1093506 (92e:46147)

[18] K. Lermer, The Grothendieck-Pietsch domination principle for nonlinear summing integral operators, Studia Math. 129 (1998), 97-112. MR1608150 (99k:47178)

[19] J. Lindenstrauss and A. Pełczyński, Absolutely summing operators in $\mathcal{L}_{p}$-spaces and their applications, Studia Math. 29 (1968), 275-326. MR0231188 (37:6743)

[20] J. Lindenstrauss, L. Tzafriri, Classical Banach Spaces. I and II, Springer-Verlag, BerlinNew York, 1977, 1979. MR0500056 (58:17766) MR0540367(81c:46001) 
[21] M. C. Matos, Fully absolutely summing and Hilbert-Schmidt multilinear mappings, Collect. Math. 54 (2003), 111-136. MR1995136 (2004e:46052)

[22] M. C. Matos and D. Pellegrino, Fully summing mappings between Banach spaces, Studia Math. 178 (2007), 47-61. MR2282489 (2007j:46117)

[23] G. Muñoz, Y. Sarantopoulos and A. Tonge, Complexifications of real Banach spaces, polynomials and multilinear maps, Studia Math. 134 (1999), 1-33. MR.1688213 (2000g:46009)

[24] G. Muñoz, Complexifications of polynomials and multilinear maps on real Banach spaces, Lecture Notes in Pure and Appl. Math, 213, Marcel Dekker, 2000, 389-406. MR1772140 (2001f:46070)

[25] L. Nachbin, Topology on spaces of holomorphic mappings, Springer-Verlag, New York, 1969. MR0254579(40:7787)

[26] D. Pellegrino, Cotype and absolutely summing homogeneous polynomials in $L_{p}$ spaces, Studia Math. 157 (2003), 121-131. MR.1980709 (2004f:46019)

[27] D. Pellegrino and M. L. V. Souza, Fully summing multilinear and holomorphic mappings into Hilbert spaces, Math. Nachr. 278 (2005), 877-887. MR2141964(2005m:46072)

[28] D. Pérez-García, Operadores multilineales absolutamente sumantes, Doctoral Thesis, Universidad Complutense de Madrid, 2003.

[29] D. Pérez-García, The inclusion theorem for multiple summing operators, Studia Math. 165 (2004), 275-290. MR2110152 (2005i:47107)

[30] D. Pérez-García and I. Villanueva, Multiple summing operators on Banach spaces, J. Math. Anal. Appl. 285 (2003), 86-96. MR2000141 (2004m:47039)

[31] D. Pérez-García and I. Villanueva, Multiple summing operators on $C(K)$ spaces, Ark. Mat. 42 (2004), 153-171. MR2056549 (2005g:47003)

[32] A. Pietsch, Absolut p-summierende Abbildungen in normierten Räumen, Studia Math. 28 (1966/1967), 333-353. MR0216328 (35:7162)

[33] A. Pietsch, Ideals of multilinear functionals, Proceedings of the Second International Conference on Operator Algebras, Ideals and Their Applications in Theoretical Physics, 185-199, Teubner-Texte Math., vol 67, Teubner, Leipzig, 1983. MR763541

[34] M. S. Ramanujan and E. Schock, Operator ideals and spaces of bilinear operators, Linear and Multilinear Algebra 18 (1985), 307-318. MR826681(87j:47062)

[35] M. L. V. Souza, Aplicações multilineares completamente absolutamente somantes, Doctoral Thesis, Unicamp, 2003.

[36] M. Talagrand, Cotype and (q,1)-summing norm in a Banach space, Invent. Math. 110 (1992), 545-556. MR1189490 (93k:46015)

Faculdade de Matemática, Universidade Federal de Uberlândia, 38.400-902, UberLÂNDIA, BRAZIL

E-mail address: botelho@ufu.br

Institute of Mathematics, University of Potsdam, 14469, Potsdam, Germany

E-mail address: braunss@rz.uni-potsdam.de

Institute of Mathematics, University of Potsdam, 14469, Potsdam, Germany

E-mail address: junek@rz.uni-potsdam.de

Departamento de Matemática, Universidade Federal da Paraíba, 58051-900, J. Pessoa, PB, BRAZIL

E-mail address: pellegrino.math@gmail.com 